A. Pramesh Rao, G. Swarup and Gopal-Krishna, eds.

\title{
Diffuse Sources in Clusters: What Turns them on?
}

\author{
Tara Murphy and R.W. Hunstead \\ School of Physics, University of Sydney, NSW 2006, Australia
}

\begin{abstract}
To investigate the rate of occurrence and possible triggering mechanisms for diffuse radio sources, we have carried out a statistical study of a volume-limited sample of 92 clusters of galaxies.
\end{abstract}

\section{Introduction}

Diffuse radio sources in clusters of galaxies - usually referred to as radio halos or relics - are characterised by low surface brightness, large extent $\sim 1 \mathrm{Mpc}$, and a steep spectral index, typically $\alpha<-1\left(S \propto \nu^{\alpha}\right)$. Diffuse sources are rare, with only $\sim 30$ known to date, and only $\sim 10$ studied extensively.

The most significant problem in identifying diffuse sources is that they are not well characterised. We based our search on the radio morphologies in the literature, keeping the constraints as broad as possible. The sample was a volume-limited subset of 92 clusters, drawn from the southern Abell, Corwin \& Olowin (1989) catalogue, and observed at $843 \mathrm{MHz}$ with the Molonglo Observatory Synthesis Telescope (MOST; Robertson 1991). The MOST, with its continuous $u v$ coverage, is well suited to detecting low surface brightness emission. The search yielded 14 candidates, including a second example of a double relic similar to A3667 (Röttgering et al. 1997); a full report will be published elsewhere.

\section{A Case Study: A3266}

A3266 is a rich cluster of galaxies and one of the first bright X-ray sources discovered in the southern sky. Its high velocity dispersion and elongated X-ray morphology suggest that the cluster is undergoing a merger. The MOST image of A3266 shows that it has a higher than average number of cluster radio sources, several with interesting morphologies. Of particular interest are the two diffuse sources shown in Fig. 1. The one to the north has an unusually steep $(\alpha \sim-2)$ spectrum which steepens with distance from the putative host galaxy. The low surface brightness source to the south was only convincingly revealed with the higher S/N of a co-added MOST image; it has no plausible optical counterpart.

\section{What Causes Diffuse Emission}

The present classification of diffuse sources as 'halos' and 'relics' has been based on their radio morphologies, projected position in the cluster, and polarization. 


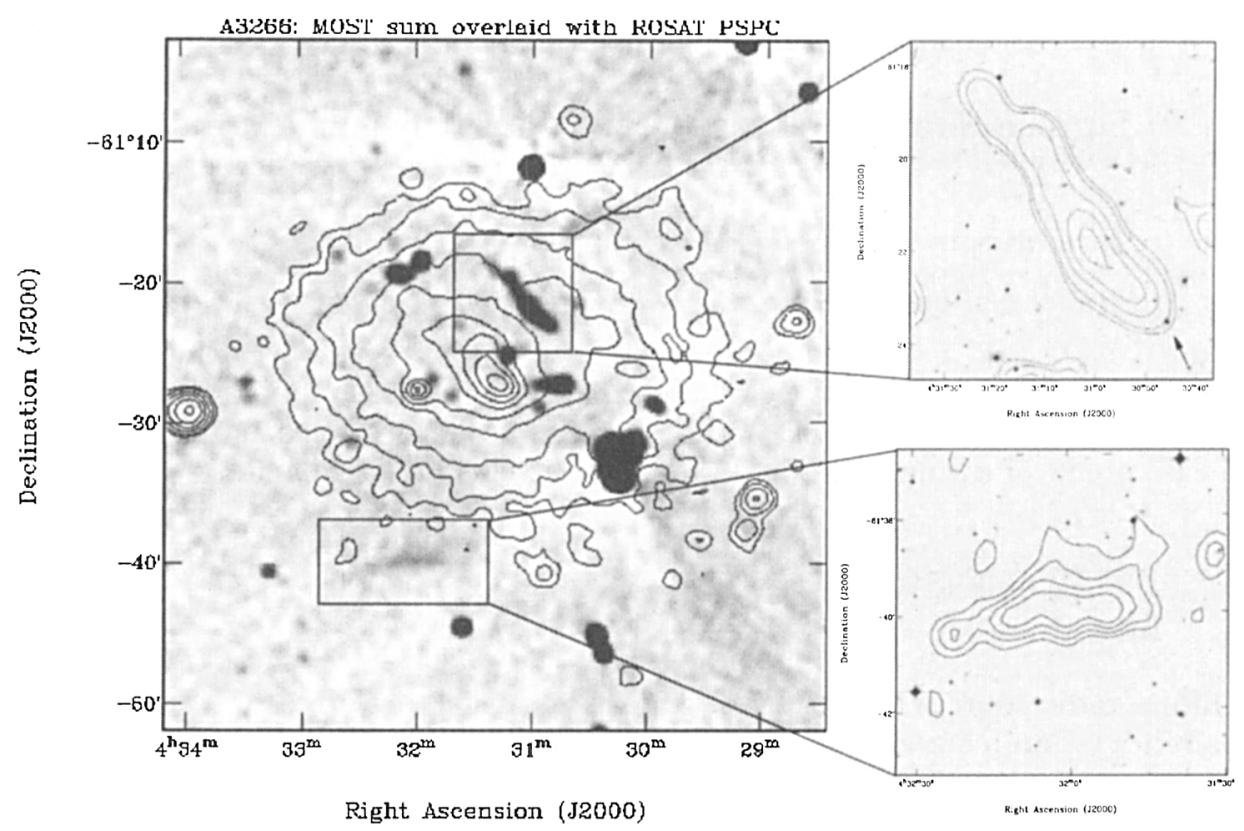

Figure 1. MOST greyscale image of A3266 overlaid with ROSAT PSPC contours. The insets show the two diffuse sources (DSS optical images overlaid with MOST contours); in the upper inset, the possible host galaxy is marked with an arrow.

We propose that these criteria are not sufficient to distinguish between the two types of sources, because of orientation selection effects. Ideally, diffuse sources should be classified on the basis of the physical processes that cause them.

The northern diffuse source in A3266 (Fig. 1), and others like it, are probably 'genuine' relic radio galaxies, which are characterised by exceptionally steep spectral indices (eg. Slee \& Reynolds, 1984). On the other hand, the most plausible explanation for sources of the type seen in A3667 is shock acceleration due to a cluster merger. Far from being old fading remains, such sources could be the products of the most powerful dynamic events in the universe.

Acknowledgments. The MOST is operated with the support of the Australian Research Council and the Science Foundation for Physics within the University of Sydney.

\section{References}

Abell, G. O., Corwin, H. G., \& Olowin, R. P. 1989, ApJS, 70, 1

Robertson, J. G. 1991, Aust. J. Phys., 44, 729

Röttgering, H. J. A., Wieringa M. H., Hunstead R. W., \& Ekers R. D. 1997, MNRAS, 290, 577

Slee, O. B., \& Reynolds, J. E. 1984, PASA, 5, 516 